\title{
Nasopharyngeal Carcinoma Incidence in North Tunisia: Negative Trends in Adults but not Adolescents, 1994-2006
}

\author{
Ben Ayoub Hizem Wided ${ }^{1 *}$, Boussen Hamouda ${ }^{2}$, Hsairi Hamadi ${ }^{3}$, Ben Abdallah \\ Mansour $^{1}$
}

\begin{abstract}
Nasopharyngeal carcinoma (NPC) is the second most common neoplasm of head and neck in Tunisia. The distribution is bimodal with a first period occurrence between 15 and 20 years old and a second peak at around 50 years of age. Undifferentiated carcinoma of nasopharynx type III (UCNT) is the predominant histological type $(\mathbf{9 3 . 4 \%})$. Data of cancer registry of North Tunisia confirmed that it is an intermediate risk area for NPC with overall ASRs of 3.6 and 1.6/100,000 respectively in males and females. This study aimed to present the evolution of incidence rate of nasopharyngeal carcinoma over a period of 12 years (1994-2006). Data of cancer registry of North Tunisia (NTCR), covering half of the Tunisian population, were used to determine evolution of NPC incidence, calculated by 5 year periods. The estimated annual percentage change (EAPC) was used as an estimate of the trend. To best summarize the behavior or the data trend across years, we used a join-point regression program. Between 1994 and 2006, we observed negative annual average change of standardized incidence in men and women $(-3.3 \%$ and $-2.7 \%)$ also for the standardized incidences which showed a rather important decline $(26.4 \%$ in males and $22.3 \%$ in females). The truncated age standardized incidence rate of NPC in adults aged of 30 years old and more $(\mathrm{N}=1209)$ decreased by $-\mathbf{- 0 . 4 \%}$ per year from 1994 to 2006 over time in north Tunisia dropping from 6.09 to 4.14 person-years. However, the rate was relatively stable during this period among youths aged $0-29$ years $(\mathrm{N}=233)$ in both sexes. NPC demonstrated a favorable evolution from 1994-2006 probably due to a improvement in socioeconomic conditions.
\end{abstract}

Keywords: Nasopharyngeal carcinoma - incidence - trends - North Tunisia

Asian Pac J Cancer Prev, 16 (7), 2653-2657

\section{Introduction}

The incidence of nasopharyngeal carcinoma (NPC) has a rather distinct and geographically well-defined distribution worldwide. High to intermediate rate are observed in certain populations and regions in China, Southeast Asia, Northern Africa, among Inuit population of Alaska, Greenland, and Northern Canada; and in migrants of Chinese and Filipino descent (Yu MC et al., 2006). Outside of these endemic areas, however, NPC can be considered a rather rare neoplasm. Incidence rates in high-risk area (Hong Kong) were between 30 and 60 times those in equivalent national or regional registry population in low- risk regions (Colombia, The United States, Finland, Zimbabwe, India and Japan over the period 1998-2002 (Curado MP et al., 2007).

Tunisia, a Northern African country, is an intermediate risk area with an incidence rates of 3,4 and 1,6/100 000 person-years respectively in men and female. NPC is the second neoplasm of head and neck cancer after laryngeal cancer with a bimodal distribution characterized by 2 peaks at 15-20 years old and a second at 50 years old (Cancer Registry Northern Tunisia: Data 1999-2003,2009; Ellouz et al., 1978).The etiology of NPC, described by several experts as enigmatic, has viral, environmental, and genetic components. (Tsao et al., 2014). An investigation of the trends and the age -incidence curves of NPC can be informative. Our paper aims to report the trends of NPC for North Tunisia in terms of NPC incidence and age -incidence profiles. Using data from the population -based North Tunisia Cancer Registry (NTCR) for the period 1994-2006. (Cancer Registry Northern Tunisia: Data 2004-2006, 2012.)

\section{Materials and Methods}

\section{Cancer registry of the north of Tunisia}

The North Tunisia Cancer Registry (NTCR), created in 1997 , covers a population of 4.436 .000 inhabitants, representing $40 \%$ of the Tunisian population, covering an area of $28,162 \mathrm{~km}^{2}$.Children represented in term of demography $24 \%$ of the total population (2005 estimation)

${ }^{1}$ Department of Epidemiology and Biostatistics, Salah Azaiez Institute of Oncology Cancer Registry North of Tunisia, ${ }^{2}$ Medical Oncology Department, Abderrahmen Mami Hospital, ${ }^{3}$ National Institute of Public Health, Tunis, Tunisia * *For correspondence: wided.benayoub@rns.tn 
and the rate of annual average population increase is estimated at $1.4 \%$. Population data (censuses 1994 and 2004) and estimates for other years were provided by the National Institute of Statistics (INS).

We used for NTCR the International Classification of Diseases for Oncology (ICD-O). To ensure comparability of RCNT results with other records, the team was trained at the International Agency for Research on Cancer (IARC) recommendations concerning the definitions of multiple primary tumors incidence and followed them

We performed comparison with Western countries (high risk) and other geographically close and / or culturally (Algeria, Kuwait, ...) by using standardized incidence rates (ASR) for age, by the direct method of Segi in 1960 and modified by Doll et al in 1966 (Doll et al., 1976).

Cases are collected with a public/private extended network in oncology academic hospitals, general hospitals and private clinics.

\section{Statistical analysis}

Incidence was calculated by 5 years periods. To adjust for the effect of difference in age we used the direct standardization according to the World Standard Population (1966) (Doll et al., 1976).

The estimated Annual Percentage Change (EAPC) was used as an estimate of the trend. To best summarize the behavior or the data trend across years, we used a Join-point Regression Program (Kim et al., 2000). It's a Windows-based statistical software package that computes and analyzes non-linear, piecewise trends of time series (e.g. time series of cancer rates). Using calendar year as a regression variable, a regression line was fitted to the natural logarithm of the rates, i.e. $y=I n$ (rate) and $\mathrm{x}=$ calendar year. EAPC was calculated using the equation $\mathrm{EAPC}=100 \mathrm{x}(\mathrm{em}-1)$. Testing the hypothesis that the EAPC is equal to zero is equivalent to testing the hypothesis that the slope of the regression line is zero, using the t-distribution of $\mathrm{m} / \mathrm{SEm}$. The number of degrees of freedom equals the number of calendar years minus 2 . The standard error of m,i.e. SEm , is obtained from the fit of the regression line. This calculation assumes that the rates increased/decreased at the entire period.

\section{Results}

In the NTCR, age-standardize incidence for NPC decreased from 4,2 (404 cases) to 3,5 (283 cases ) per 100000 person - year between 1994-1998 and 2002-2006 among men and from 1,8 to 1,3 per 100000 person - year among women in the corresponding time periods. We continue to observe in the Tunisian patient a first peak at "15-20" year old and, a subsequent decline in risk of up to the ages 30-39 years, followed by increasing risk with age to a second peak. Around 50 years. This disease mainly affects the man with a sex ratio of 2.1. The average age at diagnosis remained higher in men than in women (48.3 years versus 43.4 years). UCNT (undifferentiated Carcinoma of Nasopharyngeal Type) remains by far the most common (93.4\%) predominant histological type in men and female and $5.8 \%$ of moderately differentiated squamous cell carcinomas with keratin production (type I) or non keratinizing (type II). Compared to the period 1994-1998, the percentage of children increased from $4.8 \%$ to $1.8 \%$. The join-point analysis confirmed the decrease evaluated by $-1.8 \%$ per year among male for the 13-year period of 1994 through 2006. In female this rate is at $-1.4 \%$ per year for this same time period. Since only one straight line is displayed (see Figures 1), one can interpret the 1994-2006 trend for male and female age-adjusted cancer incidence rates as being constant (in this case, consistently on the decrease) throughout the 13year period being studied. In both sexes, the truncated age standardized incidence rate of NPC in adults aged of 30 years old and more $(\mathrm{N}=1209)$ decreased by $-0.4 \%$ (not significant) per year from 1994 to 2006 over time in north

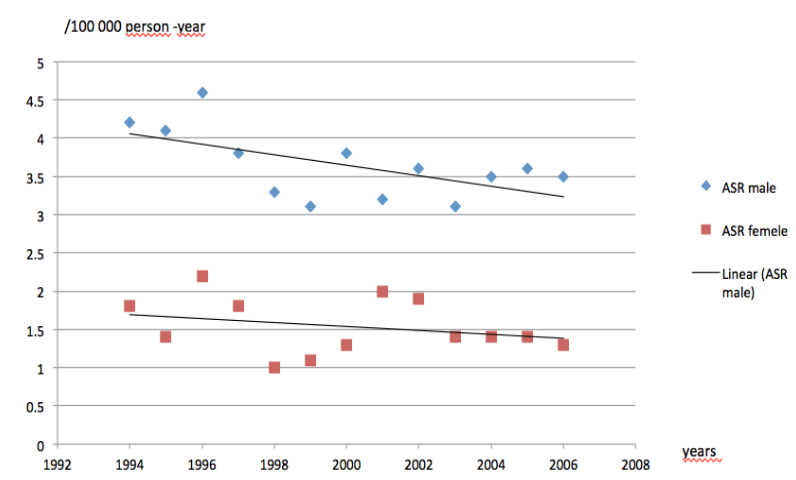

Figure 1. Males and Female Age-adjusted Incidence Rates

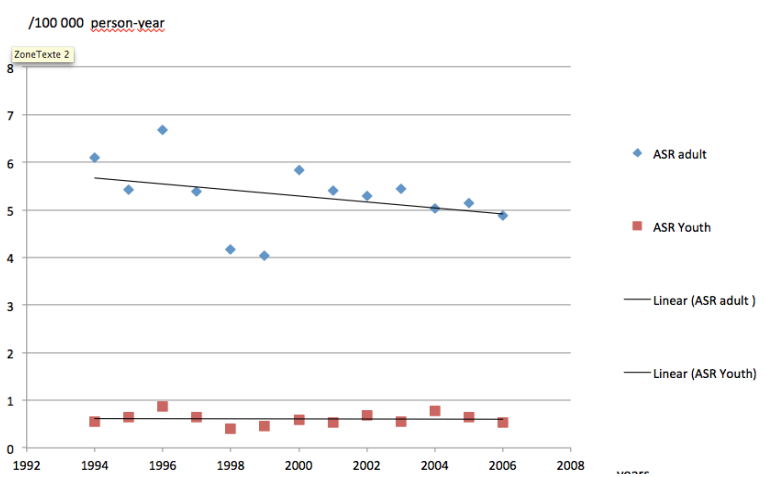

Figure 2. Truncated Incidence Rates among Youth (< 30 Years Old) and Adult (>or=to 30 years old )

Table 1. Truncated Age Standardized Incidence Rate of NPC in Adult and Child in North of Tunisia

\begin{tabular}{|c|c|c|c|c|c|c|}
\hline & \multicolumn{3}{|c|}{ Both sexes $\geq 30$} & \multicolumn{3}{|c|}{ Both sexes $<30$} \\
\hline & ASR* & $\mathrm{N} * *$ & $\overline{\text { population }}$ & $\overline{\mathrm{ASR} *}$ & $\mathrm{~N}^{* *}$ & population \\
\hline 1994 & 6.09 & 91 & 1670900 & 0.55 & 16 & 2609200 \\
\hline 1995 & 5.42 & 83 & 1783700 & 0.65 & 20 & 2642900 \\
\hline 1996 & 6.68 & 103 & 1751500 & 0.88 & 25 & 2658500 \\
\hline 1997 & 5.38 & 83 & 1806600 & 0.65 & 21 & 2664600 \\
\hline 1998 & 4.16 & 70 & 1876600 & 0.41 & 12 & 2659200 \\
\hline 1999 & 4.04 & 71 & 1956700 & 0.45 & 14 & 2626700 \\
\hline 2000 & 5.83 & 84 & 2010200 & 0.59 & 19 & 2636800 \\
\hline 2001 & 5.4 & 97 & 2061200 & 0.53 & 17 & 2628400 \\
\hline 2002 & 5.3 & 102 & 2116500 & 0.68 & 24 & 2621000 \\
\hline 2003 & 4.45 & 91 & 2203800 & 0.55 & 17 & 2602600 \\
\hline 2004 & 5.03 & 107 & 2252800 & 0.77 & 22 & 2597300 \\
\hline 2005 & 5.14 & 106 & 2304200 & 0.64 & 21 & 2592400 \\
\hline 2006 & 4.87 & 105 & 2358200 & 0.53 & 16 & 2584100 \\
\hline
\end{tabular}

* Age-adjusted incidence rate ** number of cases per year 
Tunisia dropping from 6.09 to 4.14 person-years figure 2).However, the rate were relatively stable during this period among youth aged of $0-29$ years old $(\mathrm{N}=233)$ in both sex despite a not significant negative Annual Percent Change (-0.1\%) (Figures 2 and Table 1).

\section{Discussion}

We reported in our population registry of North Tunisia a significant decrease of incidence of NPC in our country, evaluated at around $1 \%$ during a period of 13 years. NPC have worldwide a geographic particular repartition with 3 distinct endemic areas: an area with very high frequency in southern China (Canton), where the incidence is 30 to 80/100 000/year (9 Time trends and age-period-cohort analyses on incidence rates of (Xie et al., 2012); Huan et al., 2012). An area of intermediate frequency (8 to 12/100 000/year) in Taiwan, Vietnam, Thailand, Malaysia, the Philippines, the Caribbean, the Mediterranean (Maghreb and Middle East), Alaska and Greenland (Bray et al .2008). And finally an area of low frequency in North China, Europe and the United States (0.5 to 2/100 000/ year) (Busson et al., 2004 ).

The male/female ratio trends to vary uniformly from 2 to 3 (Bray and al., 2008).Within the 3 NPC histological subtypes, type I the differentiated form, is more common in low-incidence populations and non familial cases (Levine et al., 1992), associated with an older age at diagnosis and an association with alcohol and cigarette consumption (Arnold et al., 2013), The Non -Keratinizing type (type III) accounted for $84.6 \%$ of all NPC new cases in Zhongshan, a high risk area and there was no obvious changing in pathological proportion over 38 years. The pathway for type III tumors, common in high-intermediate risk populations, includes an initial genetic polymorphism (Bray et al., 2008) associated with chronic (dietary) exposure to nitrosamine compound (Tsao et al., 2014). EBV infection is also considered a critical step in the pathway of undifferentiated malignancies (Tsao et al., 2014). In intermediate to high risk Chinese populations, consumption of salted fish, especially during weaning in childhood, is associated with elevated risk of NPC (Tsao et al., 2014).

In North African countries, such as Tunisia-AlgeriaMorocco, case-witness studies, suggested that salted foods prolonged consumption, like quaddid (dried mutton stored in oil), harissa (spicy salted sauce, rancid butter and khelii (dried meat, salty, spicy, cooked and preserved in a mixture of oil and melted bovine greases) is associated with increased NPC risk (Feng et al., 2007) Other environmental factors such as tobacco, cannabis and domestic cooking fumes intake (cooking in the main living room) during childhood, have recently been described as a risk factors for NPC in North African countries as in Tunisia (Feng et al., 2009). We're always observing in North Tunisia a bimodal distribution characterized by 2 peaks at 15 - 20 years old and a second at 50 years old, previously reported in several intermediate- risk populations in Northern Africa (Tabyaoui et al., 2013) and Western Asia (Andejani et al., 2004; Barista et al., 2007; Tsao et al., 2014; ). The bimodality of disease rates, suggests the possibility of distinct causal entities. The early peak in incidence, although unexplained, suggests a role for major susceptibility genes in the pathogenesis, such as certain alleles of the human leukocyte antigen classes I and II. Human leukocyte antigen (Okni-Baizig et al., 2001; Li X et al., 2007; Makni et al., 2010) possibly mediated by other factors including EBV infection (AHadhriGuiga et al., 2006; Yadi et al., 2007) for which age at infection may be critical (Bray et al., 2008). The second peak in low-incidence populations may owe more to the classic risk factors for NPC, heavy smoking, high alcohol consumption, and possibly long-term exposure to certain occupational carcinogens (Bray et al., 2008) according to the same study the observed curves in high-risk populations are unimodal, although a masking of the first peak via early epigenetic events is a possible explanation for the lack of bimodality (Bray et al., 2008). The first incidence decrease arose from Singapore, among Chinese population in both sexes, with a peak and subsequent decline beginning in the rate of the age group 45-49 years. This decline with age has been postulated as compatible with either a viral or carcinogenic exposure early in life, or an exhaustion of the pool of genetically susceptible individuals (Bray et al., 2008, Tsao et al., 2014). The low risk population curves shown in the same study convey a systemic pattern of continual increase in risk by age up to a first peak in late adolescence/early adulthood (ages 15-24 years), a subsequent decline in risk of up to the ages 30-39 years, followed by increasing risk with age to a second peak later in life (ages 65-79 years). A recent Chinese study from the high incidence area of Guandzu, reported similar results over the time period of 2000-2011 with a peak incidence occurred in the 50- to 59-year age group, The incidence rates was $-3.26 \%$ (95\%CI: $-5.4 \%$ $-1.1)$ for males and $-5.74 \%$ (95\% CI: $-8.9 \%--2.5)$ for females, resulting in a total decrease of $39.3 \%$ (from 22.14 to 13.44 per 100,000 population) for males and $48.6 \%$ (from 10.1 to 5.18 per 100,000 population) for females over this 12-year period. The AAPCs in NPC mortality rates were $-4.62 \%(95 \% \mathrm{CI}:-3.5 \%--5.7)$ for males and $-6.75 \%(95 \%$ CI: $-5.2 \%-8.3)$ for females, resulting in a total decrease of $-46.1 \%$ (from 12.1 to 6.54 per 100,000 population) for males and $51.7 \%$ for females ( $\mathrm{Li}$ et al., 2014). In NTCR for the period 1994-2006, We observed a decreased of the age-standardize incidence from 4,2 (404 cases ) to 3,5 (283 cases )/ 100000 between 1994-1998 and 2002-2006 among men and from 1,8 to 1,3/100 000 among female in the corresponding time periods. In both sexes, the truncated age standardized incidence rate of NPC in adults aged of 30 years old and more decreased by $-0.4 \%$ per year from 1994 to 2006 ,however, the rate were relatively stable during this period among youth aged of 0-29 years old in both sex despite a not significant negative Annual Percent Change (-0.1\%). While the incidence rate of NPC were relatively stable among Singapore Chinese of both sexes over the 20-years period between 1973 and 1992, there were an approximately 15 percent drop in incidence in men and 30 percent drop in incidence in female during 1993-1997 (Parkin et al., 1997). Many studies have reported increased risks associated with certain foods eaten in high-risk areas including salted fish, 
various preserved foods, and hot spices, which are high in nitrosamine compounds and volatile nitrosamines (Chang et al., 2006), and it is possible that such a chronic dietary exposure over a sustained period of time may explain the age curves seen in high-risk populations. In the same study, trends restricted to NPC at younger ages $<50$ years, are generally flat in both low- and high-risk populations, although declines in both sexes are more visible in recent periods in the Singapore Chinese and Filipino populations (F Bray et al., 2008). The NPC epidemiological trends changes may be influenced by the geographic and ethnic distribution (Kuang-rong et al., 2010). Previous studies found that the incidence of NPC in southern china, as well as in other areas, display an inverse relation with socioeconomic class (Lau et al., 2013). An increase of socioeconomic level and access to early diagnostic of NPC is associated with the decline of NPC incidence (Lau et al., 2013), This decline of NPC incidence in Tunisia is probably associated with changes in NPC risk factor level .Commonly suspected risk factors for NPC in North Africa include Epstein-Barr virus (EBV) infection, environmental factors and genetic susceptibility (Laantri et al., 2011). in Tunisia Consumption of rancid butter and rancid sheep fat was associated with increased NPC risk, whereas that of cooked vegetable was protective in north Africa (Feng et al., 2007). In North Tunisia, the increase of socio economic level as well quite disappearance of salted food could explain as in south east Asia this incidence decrease. Such lifestyles changes are consistent with the observed NPC incidence decrease (Luo et al., 2007). The relatively stable risk of youth population in north Tunisia compared to adults suggest that inherited genetic predisposition also plays an important role in ontogenesis, Previous studies have shown some haplotypes of HLA system to be associated with increased risk of NPC in Tunisia (Mokni-Baizig et al., 2001; Laantri et al., 2011,Ghandri $\mathrm{N}$ et al., 2011) and in several high-risk population such as in Chinese American (F Bray et al., 2008). However, given the relatively stable genetic background of north Tunisian population, the drop of NPC incidence in adults population of the north of Tunisian suggest an important role of environmental factors on the development of NPC. However, because the smoking epidemic is ravaging the country, we should expect an incidence increase of nasopharyngeal keratinizing carcinoma. This finding is in agreement with findings from North America that cigarette smoking was significantly associated with differentiated NPC where it predominate (Feng et al., 2007). It is clear that in North -Tunisia, Probably all over the country, cancer incidence rates of NPC of both genders slightly decreased throughout the entire period (1994-2006) whereas there are a relatively stable risk of youth population .In endemic countries (Taiwan, Hong Kong ...), several authors have already noticed the same decrease in the incidence. For authors, decrease in nasopharyngeal carcinoma incidence in Taiwan is expected to continue because of westernization of lifestyle .In Tunisia, it is expected that decline continue. However, because the smoking epidemic is ravaging the country, we should expect an incidence increase of nasopharyngeal keratinizing carcinoma?
In conclusion, there are several limitations of this study. The major one is the limited sample size during periods, the study power may not be enough to detect the epidemiological change in NPC Second, our hypothesis that lifestyles and other environmental factors are the main reasons for the incidence trend of NPC was based mainly on previous case-control studies using questionnaire interview. These studies are subject to recall bias, and it's difficult to confirm a dose-response relationship or to do longitudinal follow up. Third, the small number of youth NPC cases can explain the stable NPC rate during the 13-year period, we cannot exclude the possibility that the truncated age standardized incidence rate of NPC in youth decrease also over the time.

\section{References}

Andejani AA, Kundapur V, Malaker K (2004). Age distribution of nasopharyngeal cancer in Saudi Arabia. Saudi Med J, 25, 1579-82.

Arnold M, Wildeman MA, Visser O, et al (2013). Lower mortality from nasopharyngeal cancer in The Netherlands since 1970 with differential incidence trends in histopathology. Oral Oncol, 49, 237-43

Ayadi W, Feki L, Khabir A, et al (2007). Polymorphism analysis of Epstein-Barr virus isolates of nasopharyngeal carcinoma biopsies from Tunisian patients. Virus Genes, 34, 137-45.

Barista I, Varan A, Ozyar E (2007). Bimodal age distribution in Hodgkin's disease and nasopharyngeal carcinoma. Med Hypotheses, 68, 1421.

Bray F, M Haugen, T A Moger, et al (2008). Age-incidence curves of nasopharyngeal carcinoma worldwide: bimodality in low-risk populations and aetiologic implications. Cancer Epidemiol Biomarkers Prev, 17, 9.

Busson P, Ooka T, Corbex M (2004). Carcinomes nasopharyngés associés au virus d'Epstein-Barr: De l'épidémiologie à la thérapeutique et au dépistage. M/S: Médecine Sciences, 20, 453-7.

Cancer Registry Northern Tunisia: Data 2004-2006 (2012). Salah Azaiz Institute, National Institute of Public Health and Epidemiology Research Unit of Cancer in Tunisia. Tunis. Ministry of health.

Curado MP, Edwards B, Shin H, et al (2007). Cancer Incidence in Five Continents. Vol. IX. IARC Scientific Publications No. 160. Lyon: IARC.

Doll R (1976). Comparison between Registries.Age-Standardized Rates. In: Waterhouse JAH, Muir CS, Correa P, Powell J (eds ). Cancer incidence in Five Continents, Vol. III (IARC Scientific Publications No. 15), Lyon, International Agency for Research on Cancer, 453-59.

Ellouz R, Cammoun M, Attia RB, et al (1978). Nasopharyngeal carcinoma in children and adolescents in Tunisia: clinical aspects and the paraneoplastic syndrome, IARC Sci Publ, 20, 115-29.

Feng BJ, Jalbout M, Ayoub WB, et al (2007). Dietary risk factors for nasopharyngeal carcinoma in Maghrebian countries. Int J Cancer, 121, 1550-5.

Feng BJ, Khyatti M, Ben-Ayoub W, et al (2009). Cannabis, tobacco and domestic fumes intake are associated with nasopharyngeal carcinoma in North Africa. Br J Cancer, 101, 1207-12.

Ghandri N, Gabbouj S, Farhat K, et al (2011). Association of HLA-G polymorphisms with nasopharyngeal carcinoma risk and clinical outcome. Hum Immunol, 72, 150 -8.

Hadhri-Guiga B, Khabir AM, Mokdad-Gargouri R, et al (2006). 
Various 30 and 69 bp deletion variants of the Epstein-Barr virus LMP1 may arise by homologous recombination in nasopharyngeal carcinoma of Tunisian patients. Virus Res, 115, 24-30.

Hsu C, Shen YC, Cheng CC, et al (2006). Difference in the incidence trend of nasopharyngeal and oropharyngeal carcinomas in Taiwan: implication from age-period-cohort analysis. Cancer Epidemiol Biomarkers Prev, 15, 856 - 61.

Huang TR, Zhang SW, Chen WQ, et al (2012). Trends in nasopharyngeal carcinoma mortality in China, 1973-2005. Asia Pac J Cancer Prev, 13, 2495-502.

Kim HJ, Fay MP, Feuer EJ, et al (2000). Permutation tests for join point regression with applications to cancer rates. Stat Med, 19, 335-51.

Kuang-rong W, Yong-long Y, You-ye Y, et al (2010). Epidemiolodical trends of NPC in China. Asia Pac J Cancer Prev, 11, 29-30.

Kuangrong W,Ying X, Jing L, et al(2010). No incidence trends and no change in pathological proportion of NPC in Zhongshan in 1970-2007. Asian Pac J Cancer Prev, 11, 1595-9.

Laantri N , Jalbout M, Khyatti M, et al (2011). XRCC1 and hOGG1 genes and risk of nasopharyngeal carcinoma in north african countries. Molec Carcinogenesis, 50, 732-7.

Lau HY, Leung CM, Chan YH, et al (2013).Secular trends of salted fish comsumption and Nasopharyngeal Carcinoma: a multi-jurisdiction ecological study in 8 regions from 3 continents. BMC Cancer, 13, 298.

Lee AW, Foo W, Mang O, et al (2003). Changing epidemiology of nasopharyngeal carcinoma in Hong Kong over a 20 year period (1980-99): an encouraging reduction in both incidence and mortality. Int J Cancer, 103, 680-5.

Levine PH, Pocinki AG, Madigan P, et al (1992). Familial nasopharyngeal carcinoma in patients who are not Chinese. Cancer, 70, 1024-9.

Li K, Lin GZ, Shen JC, et al (2014). Time Trends of nasopharyngeal carcinoma in urban Guangzhou over a 12-year period (2000-2011): declines in both incidence and mortality. Asian Pac J Cancer Prev, 15, 9899-903.

Li X, Ghandri N, Piancatelli D, et al (2007). Associations between HLA class I alleles and the prevalence of nasopharyngeal carcinoma (NPC) among Tunisians. J Transl Med, 5, 22.

Luo J, Chia KS, Chia SE, et al (2007). Secular trends of nasopharyngeal carcinoma incidence in Singapore, Hong Kong and Los Angeles Chinese populations, 1973-1997. Eur J Epidemiol, 22, 513-21.

Makni H, Daoud J, Ben Salah H, et al (2010). HLA association with nasopharyngeal carcinoma in southern Tunisia. $\mathrm{Mol}$ Biol Rep, 37, 2533-9.

Mokni-Baizig N, Ayed K, Ayed FB, et al (2001).Association between HLA-A/-B antigens and DRB1 alleles and nasopharyngeal carcinoma in Tunisia. Oncology, 61, 55-8.

Parkin DM, Whelan SL, Ferlay J et al (1997). Cancer incidence in five continents. Vol 7. Lyon, France IARC p334-7.

Cancer registry of North Tunisia (2009), Data of 1999-2003. ISBN : 978-9973-9798-2-7 Tunis Tunisia.

Tabyaoui I, Serhier Z, Sahraoui S, et al (2013). Immunohistochemical expression of latent membrane protein 1(LMP1) and p53 in nasopharyngeal carcinoma. Moroccan experience African Health Sciences, 13, 710-7.

Tsao SW, Yip YL, Tsang CM, et al (2014). Etiological factors of nasopharyngeal carcinoma. Oral Oncol, 50,330-8.

Xie SH, Gong J, Yang NN, et al (2012). Time trends and ageperiod-cohort analyses on incidence rates of nasopharyngeal carcinoma during 1993-2007 in Wuhan, China. Cancer Epidemiology, 36, 8-10.

Yu MC, Yuan J (2006). Nasopharyngeal cancer. In: Schottenfeld
D,Fraumeni-JF J, editors. Cancer epidemiology and prevention. 3rded. New York: Oxford University Press, 620-6. 